\title{
El control interno en base a la normativa legal una herramienta para mitigar el riesgo empresarial caso de estudio
}
Internal control based on legal regulations, a tool to mitigate business risk case study

María Del Carmen Moreno Albuja. ${ }^{1}$, Arias González Iván Patricio. ${ }^{2}$, Raquel Virginia Colcha Ortiz. ${ }^{3}$ \& Rómulo Agustín Cortez Manzano.. ${ }^{4}$

\begin{abstract}
.
DOI: https://doi.org/10.33262/visionariodigital.v4i3.1363

To research the relationship between the application of internal control and business risk is the main objective of the investigation. The research has been developed using the direct intervention method using internal control questionnaires, once the level of risk and confidence is defined, the type of relationship between the study variables is determined, which has made it possible to determine that the average risk level in the eight component evaluated it is equivalent to $56.63 \%$ and the level of confidence to $43.37 \%$, in addition to an operative risk higher than $100 \%$.

This research becomes a tool for timely decision making by "Cooperativa de Ahorro y Crédito Sumac Llacta.Ltda”, to implement internal control and mitigate business risks.
\end{abstract}

Keywords: Internal control, risk, decision making.

\footnotetext{
${ }^{1}$ Escuela Superior Politécnica de Chimborazo, Facultad de administración de empresas, Riobamba, Ecuador carmen.moreno@espoch.edu.ec

${ }^{2}$ Universidad Nacional De Chimborazo, Facultad de ciencias políticas y administrativas, Riobamba, Ecuador, ivan.arias@unach.edu.ec

${ }^{3}$ Escuela Superior Politécnica de Chimborazo, Facultad de Administración de Empresas, Riobamba, Ecuador, raquel.colcha@espoch.edu.ec

4 Agencia de Regulación y Control de las Telecomunicaciones, Director Zonal 3, Riobamba, Ecuador, agustin2007.cortez@gmail.com
} 


\section{Resumen.}

Investigar la relación existente entre la aplicación del control interno y el riesgo empresarial es el principal objetivo de la investigación. La investigación se ha desarrollado mediante el método de intervención directa utilizando cuestionarios de control interno, una vez definido el nivel de riesgo y confianza se determina el tipo de relación existente entre las variables de estudio, lo cual ha permitido determinar que el nivel de riesgo promedio en los ocho componentes evaludos equivale al $56.63 \%$ y el nivel de confianza al $43.37 \%$, además de un riesgo operativo superior al $100 \%$.

La presente investigación se convierte en una herramienta para la toma de decisiones oportunas por parte de la "Cooperativa de Ahorro y Crédito Sumac Llacta Ltda", para implementar el control interno y poder mitigar los riesgos empresariales.

Palabras claves: Control interno, riesgo, toma de decisiones.

\section{Introducción.}

El control interno y el riesgo empresarial son fundamentalmente importantes para el bienestar de una organización, para el caso de estudio una entidad financiera, a través del control interno se busca determinar el riesgo empresarial que afectará a la Coopetativa de Ahorro y Crédito Sumac Llacta Ltda, una vez determinado el riesgo, se propondrá un plan de mejoras para minimizar el riesgo.

A través de la investigación se busca determinar que el control interno es una herramienta útil para mitigar los riesgos a los cuales se expone la organización, por lo que se desarrolló el estado del arte de las variables de estudio.

\section{El control interno.}

El control interno representa un procedimiento organizacional que certifique a los activos de la entidad se hallen apropiadamente resguardados, los registros contables son indiscutibles y la están debidamente protegidos, los registros contables son fehacientes y los movimientos organizacionales se ejecutan de manera eficiente acorde a los lineamientos establecidos por la dirección de la entidad.

Según la legislación ecuatoriana, control interno se define como un asunto integral ejercido por la máxima autoridad, dirección y personal de cada entidad, que proporciona seguridad razonable para lograr metas empresariales establecidas, así como la protección de recursos económicos. Este tipo de control está orientado a cumplir con la codificación jurídica, técnica, administrativa promoviendo eficiencia y eficacia en las operaciones de la organización, avalando la seguridad y congruencia de la información, así como la adopción de las medidas oportunas para corregir las deficiencias de control. 
Desde un enfoque financiero, el control interno representa una escala de herramientas de gestión enfocadas a objetivos generales que certifiquen el cumplimiento de la normativa a la cual se rigen las empresas tanto a nivel privado como público, así como la fiabilidad de datos e información financiera empresarial y facilitar tanto la eficiencia como la efectividad de las operaciones de la entidad.

Con lo antes expuesto el control interno está diseñado para asegurar que las operaciones realizadas por la organización cumplen con estándares básicos en varias áreas como por ejemplo protección contra el mal uso o uso ineficiente de los recursos financieros y humanos, salvaguardar activos, establecer planes de gastos, lucha contra el fraude y el error y mantener registros contables satisfactorio que permitan a la organización elaborar informes financieros y de gestión fiables de manera oportuna.

Un adecuado control interno permite a cualquier organización tener mayores posibilidades de alcanzar sus objetivos previamente establecidos, pudiendo reducir la acumulación y la gestión real de las ganancias y las empresas que divulgan el informe de control interno del auditor tienen menor nivel de ganancias (Fang y Jin 2011). El leve control interno en las empresas se encuentra asociado con el costo de las deudas, que en promedio el diferencial crediticio de una empresa en su deuda incrementa marginalmente si revela la materialidad (Dhaliwal et al., 2011).

\section{Objetivos del Control interno.}

De acuerdo con las expresiones (Estupiñan, 2006), El control Interno comprende el plan de organización y el conjunto de métodos y procedimientos que aseguren que los activos están debidamente protegidos, que los registros contables son fidedignos y que la actividad de la entidad se desarrolla eficazmente según las directrices marcadas por la administración"

Entre los objetivos del Control Interno podemos mencionar:

- Salvaguardar los activos de la organización evitando pérdidas por fraudes o negligencias.

- Asegurar la exactitud y veracidad de los datos contables y financieros, que son utilizados por la dirección para una adecuada toma de decisiones.

- Incentivar la eficiencia en el uso de los recursos.

- Estimular el seguimiento de las prácticas decretadas por la gerencia.

- Promover, evaluar y velar por la seguridad, calidad la mejora continua de todos los procesos en general.

- Adoptar medidas de protección para los activos físicos en forma adecuada.

- Determinar lineamientos para evitar que se realicen procesos sin las adecuadas autorizaciones.

- Generar una cultura de control en todos los niveles de la organización. (Paz, 2012) 


\section{Beneficios de utilizar coso en el control interno.}

Según (Paz, 2012). Algunos de los beneficios de utilizar el estándar COSO en las organizaciones son:

- Promueve la gestión de riesgos en todos los niveles de la organización y establece directrices para la toma de decisiones de los directivos para el control de los riesgos y la asignación de responsabilidades.

- Ayuda a la integración de los sistemas de gestión de riesgos con otros sistemas que la organización tenga implantados.

- Ayuda a la optimización de recursos en términos de rentabilidad.

- Mejora la comunicación en la organización.

- Mejora el control interno de la organización.

\section{Tipos de control interno.}

De acuerdo con las aportaciones de (Paz, 2012), el control interno puede ser de dos tipos:

\section{- Control interno administrativo.}

Es el plan de organización que está adoptado por cada entidad, tomando en forma independiente un conjunto de procedimientos y acciones preventivas y correctivas los cuales, establecidos en forma adecuada, apoyan al logro de los objetivos administrativos como; permitir a la gerencia mantenerse informado de la administración de la empresa, coordinar funciones del personal en general, controlar el logro de los objetivos establecidos, definir que los funciones se estén ejecutando en forma eficiente y determinar si la entidad y sus colaboradores están cumpliendo con las políticas establecidas. (Paz, 2012)

\section{- Control interno contable.}

Se deriva del sistema de Control Interno administrativo, de su sistema de información, ya que el Control interno contable es el que genera la base de datos de la cual se alimentará el Sistema de Información. Los principales lineamientos para que el Control interno contable sea eficiente, se debe verificar que; las operaciones sean registradas en forma integral, oportuna, con una correcta valuación, según su ocurrencia, reveladas y presentadas en forma adecuada. Todas las operaciones realizadas en la entidad deben reflejar la existencia y pertenencia, esto es verificable a través de conteos físicos y cruce de información. Estas operaciones deben ser ejecutadas únicamente con las autorizaciones de los niveles permitidos. (Paz, 2012).

\section{El riesgo empresarial.}

Las empresas existen con el fin de crear valor a sus grupos de interés, en la realización de sus actividades se enfrentan con la falta de evidencia en diversos ámbitos, razón por la cual las 
organizaciones buscan determinar el grado de incertidumbre que permita la generación de valor en forma sostenible (Ambrosone, 2007).

Actualmente las organizacionaes han sufrido cambios de manera sostenible, operativa o estructural, con el fin de dar respuesta a las necesidades del entorno económico, en el cual desarrolla sus operaciones y adaptarse a los cambios, en este sentido el riesgo representa la probabilidad de que el ente económico no logre asumir costos y gastos fijos, así como las operaciones financieras (Celaya y López. 2004).

La administración de riesgos empresariales considera escenarios en los cuales se desenvuelve cada organización, con lo antes expuesto se hace necesario identificar, medir, monitorear y dar tratamiento específico a los riesgos posibles, para admitirlos, eludirlos, transferirlos y mitigarlos (IFAC, 2009 y COSO, 2014).

Lo antes expuesto se consigue a través de diligencias de control, que atiendan sucesos de riesgo inherente al actuar con otros presentes, mismos que se podrían traducir en siniestros (Carvajal, 2014).

\section{Variables de Riesgo.}

Se consideran tres variables dentro del riesgo como son:

- Riesgo Operativo, financiero y total.

- Riesgo sobre el comportamiento de la rentabilidad.

- Riesgo del entorno o riesgo país.

Riesgo Operativo: surgen de los movimientos empresariales y su medio, en lo referente al riesgo operacional, traduce en la probabilidad de que la empresa no pueda asumir los costos y gastos fijos de operaciones vinculadas a la actividad empresarial.

$$
\text { Riesgo Operativo }=\frac{\text { Costos y gastos de operación fija }}{\text { Ingresos-Costos y Gastos Variables }}
$$

Riesgo Financiero: representa la imposibilidad de revolver gastos fijos por financiamiento

$$
\text { Riesgo Financiero }=\frac{\text { Gastos Financieros }}{\text { Utilidad de Operación }}[2]
$$

Riesgo Total: denominado riesgo estructural, en este contexto este tipo de riesgo se vincula con la probabilidad de que la empresa caso de estudio no pueda solventar costos y gastos fijos tanto operacionales como financieros.

$$
\text { RiesgoTotal }=\frac{\text { Costos Fijos operativos y financieros }}{\text { Ingresos-Costos o gastos variables }}[3]
$$


Riesgos del control interno.

- Riesgo inherente, es la susceptibilidad del saldo de una cuenta o clase de transacciones a una representación errónea que pudiera ser de importancia relativa, individualmente o cuando se agrega con representaciones erróneas, asumiendo que no hubo controles internos relacionados. (Normas Internacionales de Auditoría., 2002)

- Riesgo de control, es el riesgo de que una representación errónea que pudiera ocurrir en el control de los bienes y que pudiera ser de importancia relativa individualmente o cuando se agrega con representaciones erróneas en otras clases, no sea prevenido o detectado y corregido con oportunidad por los sistemas de contabilidad y de control interno. (Normas Internacionales de Auditoría., 2002)

- Riesgo de detección, es el riesgo de que los procedimientos sustantivos de un evaluador no detecten una representación errónea que existe en un saldo de clase de transacciones que podría ser de importancia relativa, individualmente o cuando se agrega con representaciones erróneas en otros saldos o clases. (Normas Internacionales de Auditoría., 2002)

\section{Metodología.}

La presente investigación es experimental, pues una vez definido las variables de riesgo, se propone la implementación de un plan de mejoras que sirva de herramienta de control interno prospectivo.

Para determinar la existencia de control interno se aplica un cuestionario a los miembros de la entidad y también se aplican las fórmulas de riesgo operativo, financiero y total desde un punto de vista empresarial, que permitan evaluar el impacto que tiene el uso del control interno en el riesgo de las empresas.

Se aplicó cuestionario de control interno a los ocho componentes que se detallan a continuación:

- Ambiente de Control.

- Establecimiento de Objetivos.

- Identificación de Eventos.

- Evaluación de Riesgos.

- Respuesta al Riesgo.

- Tipos de actividad de control.

- Información y comunicación.

- Actividades de monitoreo.

Los datos utilizados para la investigación provienen de los estados financieros de la organización, así como de su entorno empresarial. 


\section{Resultados.}

Tabla 1. Valoración del CI

\begin{tabular}{lcc}
\hline Componente de control interno & Nivel de riesgo & Confianza \\
\hline Ambiente de Control & $55 \%$ & $45 \%$ \\
Establecimiento de Objetivos & $52 \%$ & $48 \%$ \\
Identificación de Eventos & $55 \%$ & $45 \%$ \\
Evaluación de Riesgos & $73 \%$ & $27 \%$ \\
Respuesta al Riesgo & $70 \%$ & $30 \%$ \\
Tipos de actividad de control & $64 \%$ & $36 \%$ \\
Información y comunicación & $32 \%$ & $68 \%$ \\
Actividades de monitoreo & $52 \%$ & $48 \%$ \\
\hline
\end{tabular}

Fuente: Cooperativa de Ahorro y Crédito Sumac Llacta Ltda.

- En el ambiente de control de la COAC se evidencia que se encuentra débil al no contar con documentación de respaldo.

- Al no contar con una planificación estratégica bien estructurada hace imposible el cumplimiento de los objetivos, metas y estrategias conforme a lo establecido en la Normativa Legal.

- El nivel de confianza es bajo, la cual no permite considerando que esto lleva a la inestabilidad en el proceso del control interno de la cooperativa al corto y mediano plazo.

- En la valoración de los riesgos la COAC no cuenta con un sistema para mitigar los mismos la cooperativa no está clara en el manejo de cartera de créditos, pues en la administración de efectivo se debe tomar en cuenta dichas recomendaciones para que se implemente un cambio.

- En los resultados obtenidos dentro del componente de respuesta al riesgo no se ha considerado la importancia de mitigar dicho riesgo poniendo en peligro las observaciones económicas y financieras de acuerdo con la Normativa Legal, se debe considerar la aplicación de herramientas como el COSO.

- En el componente de información y comunicación se evidencia la comunicación continua y permanente entre directivos y empleados, así como también la socialización de los manuales de procesos.

- Dentro del componente de actividades de monitoreo, de la cooperativa se encuentra que se han tomado como referencia las normas y estatutos legales que debe ser considerados por el consejo de vigilancia para lo cual se evidencia la falta de la aplicación por parte del organismo de acuerdo con las actividades que a ellos les compete.

Dentro del control interno y el riesgo empresarial, se han obtenido los siguientes resultados, se determina que el nivel de riesgo se han analizado los ocho componentes fueron evaluados y se encuentran en un porcentaje de 56,63\% del $100 \%$ del análisis, representa un nivel medio 
de riesgo, por lo que se hace referencia al no ejecutar un sistema de control interno el nivel de riesgo se incremente.

Nivel de riesgo operativo:

$$
\begin{gathered}
\text { Riesgo Operativo }=\frac{\text { Costos y gastos de operación fija }}{\text { Ingresos }- \text { Costos y Gastos Variables }} \\
\text { Riesgo Operativo }=\frac{20000}{356890,68-338956,34} \\
\text { Riesgo Operativo }=1.11
\end{gathered}
$$

Al evidenciar el riesgo operativo de la COAC Sumac Llacta equivalente al 1.11\%, podemos deducir que se encuentra en un nivel de alto riesgo en su estructura financiera que no permiten conocer los costos y gastos fijos reales, por la falta del sistema de control interno en sus procesos.

\section{Conclusiones.}

- Se concluye que aplicando los ocho componentes de control interno se evidencia que el nivel de riesgo está valorado en un promedio de $56.63 \%$, la cual indica que está en un nivel medio.

- El nivel del riesgo operativo sobrepasa el $100 \%$, por lo que se señala anteriormente que la cooperativa tiene una difícil situación en su estructura financiera, demostrando así que la hipótesis planteada en la investigación, al no ser aplicado el control interno da paso al aumento de nivel de riesgo.

- Es esta investigación la mayor deficiencia encontrada es la valoración de los riesgos, es que la cooperativa no ha implementado sistemas de control, ya que estos son las bases fundamentales para la administración de esta, por lo que hace referencia que el riesgo empresarial aumente progresivamente en el caso de estudio se evidencia, que al aplicar sistemas de control interno se puede minimizar los riesgos de manera oportuna.

\section{Referencias bibliográficas.}

Ambrosone, M. (2007). La administración del riesgo empresarial: una responsabilidad de todos-El enfoque COSO. 
Vol. 4, $\mathrm{N}^{\circ} 3$, p. 204-213, julio-septiembre, 2020

Carvajal, A. P. L. (2014). El riesgo empresarial y su relación con las Normas Internacionales de Auditoria y Aseguramiento. Revista Civilizar de Empresa y Economía, 5(9), 88104.

Celaya Figueroa, R., \& López Parra, M. E. (2004). ¿Cómo determinar su riesgo empresarial? Revista escuela de administración de negocios, (52).

Dhaliwal D, Hogan C, Trezevant R, Wilkin M (2011) Internal control disclosures, monitoring, and the cost of debt. Account Rev 86(4):1131-1156.

Estupiñan, R. (2006). Control interno y fraudes con base a los ciclos transacionales: Análisis del informe COSO I y II, 2a ed. Bogotá: Ecoe Ediciones.

Paz, L. (2012). Capítulo I. Control interno 1.1 Definición "el término"Sistema de control Interno". Obtenido

de https://dspace.ups.edu.ec/bitstream/123456789/1450/1/CAPITULO\%201.pdf.

Normas Internacionales de Auditoría. (2002). NIA 6: Evaluación de riesgos y control interno. Sección 400. Ecuador: Ábaco. 
PARA CITAR EL ARTÍCULO INDEXADO.

Moreno Albuja, M. D. C., Iván Patricio, A. G., Colcha Ortiz, R. V., \& Cortez Manzano, R. A. (2020). El control interno en base a la normativa legal una herramienta para mitigar el riesgo empresarial caso de estudio. Visionario Digital, 4(3), 204-213. https://doi.org/10.33262/visionariodigital.v4i3.1363

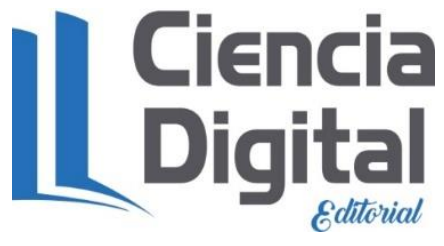

El artículo que se publica es de exclusiva responsabilidad de los autores y no necesariamente reflejan el pensamiento de la Revista Visionario Digital.

El artículo queda en propiedad de la revista y, por tanto, su publicación parcial y/o total en otro medio tiene que ser autorizado por el director de la Revista Visionario Digital.
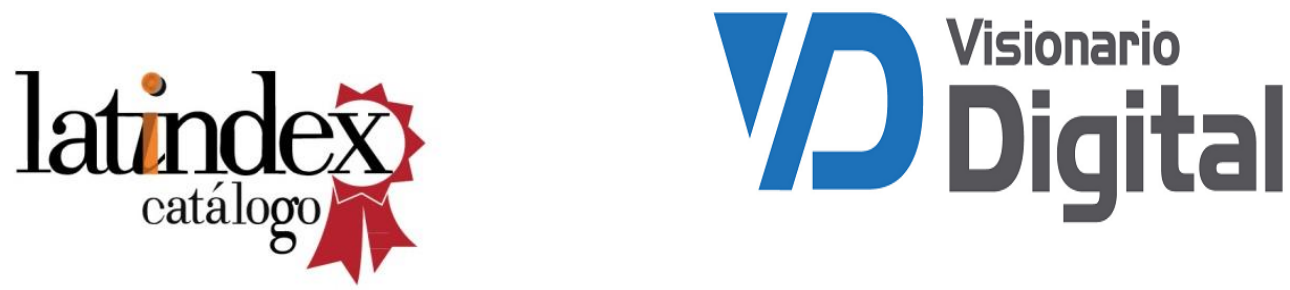